\title{
Near Invariance and Local Transience for Random Diffeomorphisms
}

\author{
Fritz Colonius \\ Institut für Mathematik, Universität Augsburg, 86135 Augsburg/Germany \\ fritz.colonius@math.uni-augsburg.de
}

and

Ale Jan Homburg

KdV Institute for Mathematics, University of Amsterdam, Plantage Muidergracht 24, 1018TV Amsterdam, The Netherlands

and

Wolfgang Kliemann

Department of Mathematics, Iowa State University, Ames, IA 50011, U.S.A.

Facultad de Matemáticas, P. Universidad Católica de Chile, Santiago, Chile

Version: July 12, 2008

For random diffeomorphisms depending on a parameter, nearly invariant sets are described via an associated deterministic control system. Conditions are provided guaranteeing that the system leaves the support of an invariant measure under small perturbations of the parameter and estimates for the exit times are given.

Key Words: Random diffeomorphisms, near invariance, local transience Subject Classification: 37H20, 93B05

\section{INTRODUCTION}

Nearly (or almost) invariant sets for random dynamical systems are subsets of the state space that are left only after long time and, maybe, are visited again after even longer times. The present paper takes up the approach developed in Colonius, Gayer, and Kliemann [8] for Markov diffusions. We develop an analogous theory for random diffeomorphisms and combine it with methods from Zmarrou and Homburg [21] for bifurcation problems based on an eigenvalue analysis of the associated Perron-Frobenius operator.

Related work includes approaches based on transfer operator theory combined with set oriented numerics in Dellnitz and Junge [11], [10], Froyland [15], Froyland and Dellnitz [16], and graph theoretic methods, Dellnitz et al. [12], as well as extensions of metastability in the classical Freidlin/Wentzell theory [14] in Schütte, Huisinga, and Meyn [20] and Bovier et al. [5], [6]; and the analysis of dominant eigenvalues of transfer operators (Schütte, Huisinga, and Deufhard [19], Deufhard et al. [13]).

The paper [8] introduces the notion of nearly invariant sets as a way to formalize the idea of almost invariance. Here the maximal amplitudes of the noise process are varied and nearly invariant sets can be described by properties of an associated deterministic control system. The escape times from a nearly invariant set become 
unbounded as the critical amplitude is approached. However, no quantitative estimates on the escape times are given. On the other hand, a loss of invariance appears to be related to a bifurcation in the considered random dynamical system, when the maximal amplitude of the noise process is considered as a bifurcation parameter. In this context [21] analyzed random diffeomorphisms and, in particular, could show that the average escape times from a neighborhood of the support of a stationary invariant measure can be estimated from below as a function of the unfolding parameter.

The present paper concentrates on the behavior of the supports of stationary measures and on exit times. The aim is to provide conditions under which the system indeed leaves the support of an invariant measure under small perturbations (note that the pertinent result in [21] on first exit times does allow infinite escape times for $\alpha$ near $\alpha_{0}$ ). For this purpose we transfer the approach from [8] to families of random diffeomorphisms depending on arbitrary bifurcation parameters and introduce the notion of local transience for nearly invariant sets. The analysis is based, as in [8], on the relations between random systems and associated deterministic control systems. In particular, we analyze loss of invariance via control sets; their invariance properties are studied here in a way which is similar to the one in Gayer [17] for fbws.

It is essential here that ergodic invariant measures have smooth densities and that the transfer operators as well as its eigenvalues and eigenspaces depend smoothly on the considered random diffeomorphisms. As shown in [21], this can be guaranteed under the assumptions stated in Section 2. Section 3 analyzes control sets, i.e., subsets of complete approximate controllability and their parameter dependence. Section 4 relates control systems to random diffeomorphisms and shows that the invariant control sets coincide with the supports of the stationary measures. In Section 5 we introduce the notions of near invariance and local transience and characterize them via the associated control system. Theorem 5 shows that invariant control sets, which lose their invariance due to a parameter change, are locally transient, nearly invariant sets. Finally, upper and lower bounds on the first exit times from these sets are given.

\section{PRELIMINARIES}

In this section we introduce families of random diffeomorphisms and cite several pertinent results from Zmarrou and Homburg [21]. Then we introduce associated discrete-time control systems.

\subsection{Random Diffeomorphisms}

The adjective smooth stands for $C^{\infty}$. Let $M$ be a smooth $d$-dimensional compact Riemannian manifold with measure $m$ induced by the Riemannian structure and let $\Delta$ be a closed domain in $d$-dimensional Euclidean space. Smoothness of a function $g$ on $\Delta$ is to be understood in the sense that $g$ can be extended to a smooth function on a neighborhood of $\Delta$. A random differentiable map is a smooth map $f: M \times \Delta \rightarrow M,(x, \omega) \mapsto f(x ; \omega)$, i.e., depending on a random parameter $\omega \in \Delta$ drawn from a measure $\nu$ on $\Delta$ with smooth density function $g: \Delta \rightarrow \mathbb{R}, \omega \mapsto g(\omega)$. A random diffeomorphism is a random map so that $x \mapsto f(x ; \omega)$ is a diffeomorphism for each $\omega$.

The following standing assumptions will be made. 
(H1) The set $\Delta$ is a domain in $\mathbb{R}^{d}$ with a piecewise smooth boundary.

(H2) The map $\omega \mapsto f(x ; \omega)$ is injective for each $x$.

(H3) For Lebesgue almost all $\omega \in \Delta$ one has $g(\omega)>0$.

From hypotheses (H1) and (H2) it follows that the number of random parameters is equal to the dimension of the state space $M$. Hypothesis (H3) implies that the support of the measure $\nu$ coincides with $\Delta$.

A smooth random map gives rise to a discrete-time Markov process through the transition functions

$$
P(x, A)=\int_{\{\omega, f(x, \omega) \in A\}} \mathrm{d} \nu(\omega) \text { for Borel sets } A .
$$

The stationary measures $\mu$ are probability measures on $M$ with

$$
\mu(A)=\int_{\operatorname{supp} \mu} P(x, A) d \mu \text { for all Borel sets } A .
$$

Consider a family $f_{\alpha}: M \times \Delta \rightarrow M, x \mapsto f_{\alpha}(x ; \omega)$, of random differentiable maps where the parameter $\alpha$ is from an interval $I$ and $f$ is continuous with respect to $(\alpha, x, \omega)$. For a sequence $\omega=\left(\omega_{0}, \omega_{1}, \omega_{2}, \ldots\right)$ let $\vartheta(\omega):=\left(\omega_{1}, \omega_{2}, ..\right)$ denote the time shift and abbreviate $f_{\alpha}^{1}(x, \omega):=f_{\alpha}\left(x, \omega_{0}\right), f_{\alpha}^{k}(x, \omega)=f_{\alpha}\left(f_{\alpha}^{k-1}(x, \omega), \vartheta^{k-1}(\omega)\right), k>$ 1. The product measure $\nu^{\infty}$ on $\Delta^{\mathbb{N}}$ is $\vartheta$-invariant.

We write $\mathcal{R}^{k}(M)$ for the space of $C^{k}$ diffeomorphisms $f$ on $M$ with $f(x ; \omega) C^{k}$ jointly in $x \in M$ and $\omega \in \Delta$ depending on a random parameter from $\Delta$ through a distribution with a $C^{k}$ density function $g$. An ergodic stationary measure $\mu$ for the smooth random map $f$ is a probability measure on $M$ such that $\mu \times \nu^{\infty}$ is an ergodic invariant measure for the skew product system

$$
S: M \times \Delta \rightarrow M \times \Delta, S(x, \omega)=\left(f\left(x, \omega_{0}\right), \vartheta(\omega)\right) .
$$

Then, see [21, Theorem 1.3], a random diffeomorphism $f \in \mathcal{R}^{\infty}(M)$ possesses a finite number of ergodic stationary measures with mutually disjoint supports. These measures are absolutely continuous with respect to Lebesgue measure with $C^{\infty}$ densities.

The following result [21, Theorem 1.13] gives an estimate from below for average escape times. For a set $W \subset M$ and $x \in W, \omega \in \Delta^{\mathbb{N}}$ let

$$
\chi_{\alpha}(x, \omega, W):=\min \left\{k \in \mathbb{N}, f_{\alpha}^{k}(x, \omega) \notin W\right\}
$$

be the first exit time from $W$ starting in $x \in W$.

THEOREM 1. Let $f_{\alpha}, \alpha \in I$, be a family of random diffeomorphisms in $\mathcal{R}^{\infty}(M)$ satisfying hypotheses (H1)-(H3) with the parameter $\alpha$ from an open interval $I$. Let $\mu_{\alpha_{0}}$ be a stationary measure of $f_{\alpha_{0}}$ for some $\alpha_{0} \in I$ and let $W$ be an open neighborhood of the support of $\mu_{\alpha_{0}}$ such that no other stationary measure has support intersecting $\mathrm{cl} W$. Then for all $k \in \mathbb{N}$ there is $C_{k}>0$ such that

$$
\int_{W} \int_{\Delta^{\mathbb{N}}} \chi_{\alpha}(x, \omega, W) \mathrm{d} \nu^{\infty}(\omega) \mathrm{d} m(x) \geq C_{k}\left|\alpha-\alpha_{0}\right|^{-k} .
$$

This result shows that the expected escape times averaged over $W$ grow faster than every polynomial. 


\subsection{Discrete-Time Control Systems}

With a random diffeomorphism $f$ depending on a parameter $\alpha$ we associate the discrete time control system (again depending on the parameter $\alpha$ )

$$
x_{n+1}=f_{\alpha}\left(x_{n}, u_{n}\right), u_{n} \in \Delta .
$$

In the following we suppress the index $\alpha$ in the notation where it is not relevant. For $u: \mathbb{N} \rightarrow \Delta$ let $\left.f^{1}(x, u):=f\left(x, u_{0}\right), f^{k}(x, u)=f\left(f^{k-1}(x, u), \vartheta^{k-1}(u)\right), u\right), k>1$. We also write $\varphi\left(k, x_{0}, u\right)=f^{k}\left(x_{0}, u\right)$. The set $\mathcal{U}$ of control functions $u: \mathbb{N} \rightarrow \Delta$ becomes a metric space by

$$
d(u, v):=\sum_{k=0}^{\infty} 2^{-k} \frac{\left\|u_{k}-v_{k}\right\|}{1+\left\|u_{k}-v_{k}\right\|} .
$$

By compactness of $\Delta$ every sequence $\left(u^{j}\right)$ of control functions contains a subsequence converging to some $u \in \mathcal{U}$. Furthermore

$$
\begin{aligned}
x^{j} & \rightarrow x \text { in } M \text { and } u^{j} \rightarrow u \text { in } \mathcal{U} \text { implies } \\
f^{k}\left(x^{j}, u^{j}\right) & \rightarrow f^{k}(x, u) \text { uniformly for bounded } k .
\end{aligned}
$$

The reachable set at time $k \in \mathbb{N}$ from $x$ is given by

$$
\mathcal{O}_{k}^{+}(x):=\left\{f^{k}(x, u), u: \mathbb{N} \rightarrow \Delta\right\}, \text { and } \mathcal{O}^{+}(x):=\bigcup_{k \in \mathbb{N}} \mathcal{O}_{k}^{+}(x) .
$$

Furthermore, define

$$
\mathcal{O}_{k}^{-}(x):=\left\{y \in M, f^{k}(y, u)=x \text { for some } u: \mathbb{N} \rightarrow \Delta\right\} .
$$

Lemma 1. Hypotheses (H1) and (H2) imply that for every $x \in M$ the map $\omega \mapsto f(x, \omega): \Delta \rightarrow M$ is continuously differentiable with $D_{\omega} f(x, \omega)$ a linear isomorphism.

Proof. This is clear, since this map is smooth and injective.

An immediate consequence of this result is that the control system is accessible, i.e. the sets $\mathcal{O}_{1}^{+}\left(x_{0}\right):=\left\{f\left(x_{0}, u\right), u \in \Delta\right\}$ and $\mathcal{O}_{1}^{-}\left(x_{0}\right):=\left\{x \in M, f(x, u)=x_{0}\right.$ for some $u \in \Delta\}$ have nonvoid interiors.

\section{CONTROL SETS}

In this section we analyze subsets of complete controllability, their boundaries, and their parameter dependence.

First we consider controllability properties of system (2) assuming from now on that the diffeomorphisms in (2) are real analytic.

Definition 1. A subset $D \subset M$ with nonvoid interior is a control set for (2) if it is a maximal set with $D \subset \mathrm{clO}^{+}(x)$ for all $x \in D$. An invariant control set is a control set $D$ with $\operatorname{cl} D=\operatorname{clO}^{+}(x)$ for all $x \in D$. The core of a control set $D$ is given by

$$
\operatorname{core} D:=\left\{x \in \operatorname{int} D, \operatorname{int}\left(\mathcal{O}^{+}(x) \cap D\right) \neq \emptyset \text { and } \operatorname{int}\left(\mathcal{O}^{-}(x) \cap D\right) \neq \emptyset\right\} .
$$


By accessibility, one finds that the closure of a control set coincides with the closure of its interior. Furthermore, a set $D \subset M$ is an invariant control set if $\operatorname{cl} D=\operatorname{clO}^{+}(x)$ for all $x \in D$ and $D$ is a maximal set with this property. We remark that, contrary to continuous time, control sets need not be connected.

Proposition 1. The number of invariant control sets in the compact state space $M$ is finite, they have nonvoid interiors, and for every $x \in M$ there exist $n \in \mathbb{N}$ and $u \in \mathcal{U}$ with $f^{n}(x, u) \in \operatorname{int} C$ for some invariant control set $C$.

Proof. This follows as in the continuous-time case (see [9]) . I

The following properties of the core have been shown by Albertini and Sontag [1, Lemma 7.3, Lemma 7.8, and Lemma 7.9], cp. also Bauer [4]; they are a consequence of analyticity.

Lemma 2. Consider a control set $D$ of the control system (2). Then core $D$ is open and

$$
\operatorname{core} D \subset \mathcal{O}^{+}(x) \text { for all } x \in D \text { and } \operatorname{cl} \operatorname{core} D=\operatorname{cl} \operatorname{int} D=\operatorname{cl} D .
$$

The time reversed system is

$$
x_{n+1}=f^{*}\left(x_{n}, u_{n}\right) \text { where } f^{*}(\cdot, v):=f^{-1}(\cdot, v), v \in \Delta .
$$

It follows easily from the definition that the control sets $D$ of the original system and the control sets $D^{*}$ of the time reversed system, are in bijective correspondence and that $\operatorname{core} D=\operatorname{core} D^{*}$.

The following result shows lower semicontinuity of control sets depending on the parameter $\alpha$, under the conditions of Section 2.1. (We note that it holds under much weaker assumptions than (H1) and (H2).)

Theorem 2. Let $D^{\alpha_{0}}$ be a control set for control system (2) $)^{\alpha_{0}}$ with $\alpha_{0} \in I$. Then there is $\delta>0$, such that for every $\alpha$ with $0<\alpha-\alpha_{0}<\delta$, there exists a unique control set $D^{\alpha}$ for (2) ${ }^{\alpha}$ with the following property: For every compact subset $K \subset \operatorname{core}\left(D^{\alpha_{0}}\right)$ there is $\delta(K)<\delta$ such that $K \subset \operatorname{core}\left(D^{\alpha}\right)$ for all $0<$ $\alpha-\alpha_{0}<\delta(K)$.

The map $\alpha \mapsto \operatorname{cl} D^{\alpha}$ is lower semicontinuous in $\alpha=\alpha_{0}$ at $\alpha_{0}$ with respect to the Hausdorff metric.

Proof. Fix a point $x \in \operatorname{core}\left(D^{\alpha_{0}}\right)$. There are $k \in \mathbb{N}$ and $u \in \mathcal{U}$ with $y=$ $f_{\alpha_{0}}^{k}(x, u) \in \operatorname{core}\left(D^{\alpha_{0}}\right)$. By the implicit function theorem applied to the map $A \times$ $\Delta^{k} \rightarrow M,(\alpha, w) \mapsto f_{\alpha}^{k}(x, w)$ there are $\delta_{x}^{1}>0$ and a neighborhood $V_{0}(y) \subset \mathcal{O}_{k}^{\alpha,+}(x)$ for all $\alpha$ with $0<\alpha-\alpha_{0}<\delta_{x}^{1}$. Since $x$ is also in the core of the control set for the time reversed system, there are $u_{1} \in \mathcal{U}$ and $l \in \mathbb{N}$ with $\varphi^{\alpha_{0}}\left(l, y, u_{1}\right)=x$. Due to continuous dependence on the parameter, there exist $0<\delta_{x}<\delta_{x}^{1}$ and an open neighborhood $V_{1}(y) \subset V_{0}(y)$, such that for every $\alpha$ with $0<\alpha-\alpha_{0}<\delta_{x}$ there is $y^{\alpha} \in V_{1}(y)$ with $f_{\alpha}^{l}\left(y^{\alpha}, u_{1}\right)=x$. Therefore it follows that $\operatorname{int} \mathcal{O}_{l}^{\alpha,-}(x) \cap V_{1}(y) \neq \emptyset$ and that $y^{\alpha}$ is contained in a certain control set $\tilde{D}^{\alpha}$ (which may depend on $x$ ). Obviously $y^{\alpha}$ is in the interior of this control set, i.e. there is a neighborhood $V\left(y^{\alpha}\right) \subset V(y)$ of $y^{\alpha}$. We would like to show that $x$ is in the interior of $\tilde{D}^{\alpha}$. There is a control $w \in \mathcal{U}$ with $f_{\alpha}^{k}(x, w)=y^{\alpha}$. Then the set $W:=\left(f_{\alpha}^{k}\right)^{-1}\left(V\left(y^{\alpha}\right), w\right) \cap$ $\varphi^{\alpha}\left(l, V\left(y^{\alpha}\right), \tilde{u}_{1}\right)$, where $\left(f_{\alpha}^{k}\right)^{-1}(\cdot, w)$ denotes the inverse mapping of $f_{\alpha}^{k}(\cdot, w)$, is a neighborhood of $x$. Obviously $W \subset \tilde{D}^{\alpha}$ and $x \in \operatorname{int} \tilde{D}^{\alpha}$. It is clear, that $\operatorname{int}\left(\mathcal{O}^{+}(x) \cap\right.$ 
$\left.\tilde{D}^{\alpha}\right) \neq \emptyset$ and $\operatorname{int}\left(\mathcal{O}^{-}(x) \cap \tilde{D}^{\alpha}\right) \neq \emptyset$, i.e. $\quad x \in \operatorname{core}\left(\tilde{D}^{\alpha}\right)$. Now fix a compact subset $K \subset \operatorname{core}\left(D^{\alpha_{0}}\right)$. Then the arguments above show that $\delta_{x}$ may be chosen independent of $x$, i.e., there is $\delta_{K}>0$ such that for all $0<\alpha-\alpha_{0}<\delta_{K}$ there is a unique control set $\tilde{D}_{K}^{\alpha}$ with the inclusion $K \subset \operatorname{core} \tilde{D}_{K}^{\alpha}$. Finally, choose a sequence of compact subsets $K_{n} \subset K_{n+1} \subset \operatorname{core} D^{\alpha_{0}}$ with $K_{0}=K$ and cl $\left[\bigcup_{n} K_{n}\right]=\operatorname{core} D^{\alpha_{0}}$. Then the control sets $D_{K_{n}}^{\alpha}$ constructed above coincide and hence the first assertion of the theorem holds with $\delta:=\delta_{K_{0}}$. Lower semicontinuity of $\alpha \mapsto \operatorname{cl} D^{\alpha}$ follows from $\operatorname{cl} \operatorname{core}\left(D^{\alpha}\right)=\operatorname{cl} D^{\alpha}$.

For continuous time systems, a control set can only be left through points on the boundary. Clearly, in the discrete time setting, points in the interior of a control set can leave this set without hitting the boundary. Nevertheless, a classification of the boundary points yields information about invariance of control sets. This result is due to Class [7]; for convenience of the reader, we include the proof.

Lemma 3. Let $D$ be a control set and assume that the control range $\Delta$ is connected. Then $D$ is an invariant control set iff $D$ is closed.

Proof. Let $x \in \operatorname{cl} D$. Then $x \in \operatorname{clO}^{+}(y)$ for all $y \in D$. Furthermore, we have $\operatorname{clO}^{+}(x) \subset \operatorname{clO}^{+}(y) \subset \operatorname{cl} D$ and $\operatorname{int} \mathcal{O}^{+}(x) \neq \emptyset$. Hence there is a point $y \in \operatorname{int} \mathcal{O}^{+}(x) \cap$ $D$. Using the definition of invariant control sets we find $\operatorname{cl} D=\operatorname{clO}^{+}(y) \subset \operatorname{clO}^{+}(x)$, i.e. $\mathrm{clO}^{+}(x)=\operatorname{cl} D$. By maximality of control sets we see that $x \in D$, hence cl $D=D$. For the converse, let $D$ be a closed control set. We have to show that for all $x \in D$ one has $\operatorname{clO}^{+}(x) \subset \operatorname{cl} D$, i.e., $\mathcal{O}^{+}(x) \subset D$. For every $y \in D$ there are $k \in \mathbb{N}$ and a control $u$ with $\varphi(k, y, u) \in \operatorname{int} D$. By continuity, there is an open neighborhood $V(y)$ with $\varphi(k, V(y), u) \subset \operatorname{int} D$. Hence there is an open set $V$ containing $D$, such that for all $z \in V$ one has $\mathcal{O}^{+}(z) \cap \operatorname{int} D \neq \emptyset$ and hence $D \subset \operatorname{cl} \mathcal{O}^{+}(z)$. Assume contrary to the assertion that there are $x \in D$ and a control $u$ with $\varphi(1, x, u) \notin D$. Since $D \subset \operatorname{cl}^{+}(x)$ there is $y \in \mathcal{O}^{+}(x) \cap D$. There is a control $v$ with $\varphi(1, x, v) \in D$. Hence $\operatorname{clO}_{1}^{+}(x) \cap D \neq \emptyset$ and $\operatorname{clO}_{1}^{+}(x) \not \subset D$. Since by assumption the control range $\Delta$ is connected, also $\mathrm{clO}_{1}^{+}(x)$ is connected, and hence there is $z \in \mathcal{O}^{+}(x) \cap(V \backslash D)$. It follows that $z \in \operatorname{clO}^{+}(y)$ for all $y \in D$ and $D \subset \operatorname{clO}^{+}(z)$ as shown above. By the definition of control sets it follows that $z \in D$ contrary to the assumption.

Definition 2. Let $D$ be a control set. Define the following subsets of the boundary $\partial D$ :

$$
\begin{aligned}
& \partial^{e x} D:=\left\{x \in \partial D, \text { there is } y \in \operatorname{int} D \text { with } x \in \mathcal{O}^{+}(y)\right\} \\
& \partial^{e n} D:=\left\{x \in \partial D, \text { there is } y \in \operatorname{int} D \text { with } y \in \mathcal{O}^{+}(x)\right\} \\
& \partial^{t g} D:=\left\{x \in \partial D, \mathcal{O}^{+}(x) \cap \operatorname{int} D=\emptyset \text { and } \mathcal{O}^{-}(x) \cap \operatorname{int} D=\emptyset\right\} .
\end{aligned}
$$

These sets are called the exit, entrance, and tangential boundaries, respectively.

Lemma 4. Assume that the control range $\Delta$ is connected. (i) The three sets $\partial^{e x} D, \partial^{e n} D$, and $\partial^{t g} D$ form a decomposition of $\partial D$. (ii) The sets $\partial^{\text {ex }} D$ and $\partial^{\text {en }} D$ are open in $\partial D$ and $\partial D \cap D=\partial^{e n} D$. In particular, $D$ is an invariant control set iff $\partial D=\partial^{e n} D$.

Proof. (i) Clearly, the union of these three sets coincides with $\partial D$ and $\partial^{t g} D$ has void intersection with $\partial^{e x} D$ and $\partial^{e n} D$. Now assume that there is $x \in \partial^{e x} D \cap \partial^{e n} D$. Then there are $y_{1}, y_{2} \in \operatorname{int} D$, times $t_{1}, t_{2} \in \mathbb{N}$ and controls $u_{1}, u_{2} \in \mathcal{U}$ such that $x=\varphi\left(t_{1}, y_{1}, u_{1}\right)$ and $y_{2}=\varphi\left(t_{2}, x, u_{2}\right)$. Due to continuity, there are neighborhoods 
$V\left(y_{1}\right) \subset \operatorname{int} D, V\left(y_{2}\right) \subset \operatorname{int} D$ and $V(x) \subset M$ such that $V(x)=\varphi\left(t_{1}, V\left(y_{1}\right), u_{1}\right)$ and $V\left(y_{2}\right)=\varphi\left(t_{2}, V(x), u_{2}\right)$. But then $V(x) \subset D$, contradicting $x \in \partial D$. (ii) Openness of $\partial^{e x} D$ and $\partial^{e n} D$ in $\partial D$ is immediate from the definitions. Similarly, the assertion $\partial D \cap D=\partial^{e n} D$ is an easy consequence of the definitions. Lemma 3 shows that the last assertion holds.

\section{STATIONARY MEASURES AND INVARIANT CONTROL SETS}

Next we proceed to describe the relations between random diffeomorphisms and control systems. We show that the supports of stationary measures coincide with the invariant control sets and that on every invariant control set there is a unique ergodic stationary measure. The proofs essentially follow the arguments in Arnold/Kliemann [2, Proposition 3.12], where the (much more complicated) continuous-time case is treated.

We continue to assume that the diffeomorphisms $f_{\alpha}$ are real analytic and that the range $\Delta$ of the noise is connected. Note that some of our results, such as the next two lemmata, hold under weaker conditions.

The following lemma shows that finite time tubes around trajectories of the associated control system have positive probability. Recall that the $n$-step transition function is defined recursively by

$$
P_{1}(x, A)=P(x, A), P_{n}(x, A):=\int_{M} P(x, d y) P_{n-1}(y, A) .
$$

Lemma 5. (i) Let $N \in \mathbb{N}$ and consider a function $u^{0}:\{0,1, \ldots, N\} \rightarrow \Delta$. Let $\nu^{N}$ be the $N$-fold product measure on $\Delta^{N}$ of $\nu$ and, for $\varepsilon>0$,

$$
V_{\varepsilon}^{N}\left(u^{0}\right):=\left\{u:\{0,1, \ldots, N\} \rightarrow \Delta,\left\|u_{k}-u_{k}^{0}\right\|<\varepsilon \text { for all } k=0,1, \ldots, N\right\} .
$$

Then $\nu^{N}\left(V_{\varepsilon}^{N}\left(u^{0}\right)\right)>0$.

(ii) Suppose that $y \in \mathcal{O}_{N}^{+}(x)$ for the associated control system. Then for every neighborhood $V(y)$ of $y$

$$
P_{N}(x, V(y))>0 .
$$

Proof. (i) This follows since the measure $\nu$ is absolutely continuous and, by hypothesis (H3) its support coincides with $\Delta$. Thus also the measure $\nu^{N}$ is absolutely continuous and its support coincides with $\Delta^{N}$.

(ii) Let $y=f^{N}\left(x, u^{0}\right)$. By continuity, there is $\varepsilon>0$ such that $f^{N}(x, u) \in V(y)$ for all $u \in V_{\varepsilon}^{N}\left(u^{0}\right)$ and $P_{N}(x, V(y))>0$ follows.

Lemma 6. For every $x \in M$ one has

$$
\operatorname{supp} P(x, \cdot)=f(x, \Delta) .
$$

Proof. The inclusion $\operatorname{supp} P(x, \cdot) \subset f(x, \Delta)$ is obvious. For the converse, let $y \in f(x, \Delta)=\mathcal{O}_{1}^{+}(x)$. By Lemma 5 (ii), $P(x, V(y))>0$ for every neighborhood of $y$ proving the assertion.

Lemma 7. For every invariant control set $C$ there exists a stationary measure $\mu$ with $\operatorname{supp} \mu \subset C$. Conversely, for every stationary measure $\mu$ the support is contained in the union of all invariant control sets. 
Proof. Recall that there are only finitely many invariant control sets $C_{1}, \ldots, C_{l}$, and these are compact. For an invariant control set $C$ the inclusion

$$
\operatorname{supp} P(x, \cdot)=f(x, \Delta) \subset C
$$

holds for every $x \in C$. Hence there exists a stationary measure $\mu$ with $\operatorname{supp} \mu \subset C$.

Conversely, let $\mu$ be a stationary measure and suppose, contrary to the assertion, that there is set $B$ with $\mu(B)>0$ and

$$
B \cap \bigcup C_{i}=\emptyset,
$$

where the union is taken over the finitely many invariant control sets $C_{i}$ in $M$. Since $\mu$ has a density, we may assume that $B \subset \operatorname{supp} \mu$ is open. Let

$$
A:=\operatorname{supp} \mu \backslash \bigcup C_{i} .
$$

Take a point $x^{0} \in B$. Then, by Proposition 1 , there are $n \in \mathbb{N}$ and $u^{0} \in \mathcal{U}$ such that $f^{n}\left(x^{0}, u^{0}\right) \in \operatorname{int} C$ for some invariant control set $C$. By Lemma 5 this implies that there is $\varepsilon>0$ with

$$
P_{n}(x, C) \geq \varepsilon \text { for all } x \in B
$$

(decreasing $B$, if necessary). Furthermore,

$$
\begin{aligned}
\mu(A) & =\int_{\operatorname{supp} \mu} P_{n+1}(x, A) d \mu=\int_{\operatorname{supp} \mu \cap \cup C_{i}} P_{n+1}(x, A) d \mu+\int_{\operatorname{supp} \mu \backslash \cup C_{i}} P_{n+1}(x, A) d \mu \\
& =\int_{A} P_{n+1}(x, A) d \mu,
\end{aligned}
$$

by invariance of the $C_{i}$. For $x \in B$ we find by the Chapman-Kolmogorov equation and using again invariance of $C$

$$
\begin{aligned}
P_{n+1}(x, A) & =\int_{M} P_{n}(x, d y) P(y, A)=\int_{M \backslash C} P_{n}(x, d y) P(y, A) \\
& \leq \int_{M \backslash C} P_{n}(x, d y) \\
& =P_{n}(x, M \backslash C)=P_{n}(x, M)-P_{n}(x, C) \\
& \leq 1-\varepsilon .
\end{aligned}
$$

This implies

$\int_{A} P_{n+1}(x, A) d \mu=\int_{A \backslash B} P_{n+1}(x, A) d \mu+\int_{B} P_{n+1}(x, A) d \mu \leq \mu(A \backslash B)+\mu(B)(1-\varepsilon)$

and we obtain the contradiction

$$
\mu(A)=\mu(A \backslash B)+\mu(B) \leq \mu(A \backslash B)+\mu(B)(1-\varepsilon)<\mu(A \backslash B)+\mu(B)=\mu(A) .
$$

Lemma 8. Let $\mu$ be a stationary measure for the random diffeomorphism $f$.

(i) If $\operatorname{supp} \mu \cap C \neq \emptyset$ for some invariant control set $C$, then $C \subset \operatorname{supp} \mu$.

(ii) If $\mu$ is ergodic, then $\operatorname{supp} \mu \cap C_{i} \neq \emptyset$ for invariant control sets $C_{i}, i=1,2$, implies $C_{1}=C_{2}$.

(iii) Suppose that $\operatorname{supp} \mu_{i} \cap C \neq \emptyset$ for ergodic measures $\mu_{i}, i=1,2$, and an invariant control set $C$. Then $\mu_{1}=\mu_{2}$. 
Proof. (i) Suppose, contrary to the assertion, there is $x \in C \backslash \operatorname{supp} \mu$. By Lemma 2 , one has $C=\mathrm{cl}$ core $C$. Hence we may assume that $x \in \operatorname{core} C$. There is an open neighborhood $V(x) \subset C$ with $V(x) \cap \operatorname{supp} \mu=\emptyset$. Pick $y \in \operatorname{supp} \mu \cap C$. Then, by Lemma 2, $x \in \operatorname{core} C \subset \mathcal{O}^{+}(y)$, and Lemma 5 shows that there is $n \in \mathbb{N}$ with

$$
P_{n}(y, V(x))>0
$$

Since the support is invariant, this is a contradiction.

(ii) This is immediate from ergodicity, since the assumption implies that $\operatorname{supp} \mu \cap$ $C_{1}$ and $\operatorname{supp} \mu \cap \bigcup_{i \neq 1} C_{i}$ are invariant sets with positive measure.

(iii) Compare Arnold/Kliemann [3].

Together, Lemmas 7 and 8 show the following theorem.

THEOREM 3. Let $\mu$ be an ergodic stationary measure for the random diffeomorphism $f$. Then the support of $\mu$ is an invariant control set of the associated control system. Conversely, for every invariant control set $C$ there exists a unique invariant measure $\mu$ with support equal to $C$.

\section{NEARLY INVARIANT SETS}

In this section, we introduce nearly invariant and locally transient sets for families of random diffeomorphisms and characterize them by properties of the associated control systems. We continue to assume that the diffeomorphisms $f_{\alpha}$ are real analytic and that the range $\Delta$ of the noise is connected.

Recall that the first exit time from a set $W \subset M$ starting in $x \in W$ is

$$
\chi_{\alpha}(x, \omega, W):=\min \left\{k \in \mathbb{N}, f_{\alpha}^{k}(x, \omega) \notin W\right\} .
$$

Definition 3. A closed set $A \subset M$ is nearly invariant for a sequence $\alpha_{n} \rightarrow \alpha_{0}$ at $x_{0} \in \operatorname{int} A$, if it has the following properties: (i) for all $n \in \mathbb{N}$ the first exit time of $x_{0}$ from $A$ satisfies $\chi_{\alpha_{n}}\left(x_{0}, \omega, A\right)<\infty$ with positive probability; (ii) for all $x \in A$ one has $\chi_{\alpha_{n}}(x, \omega, A) \rightarrow \infty$ almost surely for $n \rightarrow \infty$ (here $\chi_{\alpha_{n}}(x, \omega, A)=\infty$ is allowed) and $\chi_{\alpha_{0}}(x, \omega, A)=\infty$.

If these properties hold for all $x_{0} \in \operatorname{int} A$, we call the set $A$ nearly invariant.

Definition 4. A closed set $A \subset M$ is locally transient for a sequence $\alpha_{n} \rightarrow \alpha_{0}$ at $x_{0} \in \operatorname{int} A$, if there is a neighborhood $W$ of $A$ such that for all $n \in \mathbb{N}$ the first exit time of $x_{0}$ from $W$ satisfies $\chi_{\alpha_{n}}\left(x_{0}, \omega, W\right)<\infty$ with positive probability.

If this property holds for all $x_{0} \in \operatorname{int} A$, we call the set $A$ locally transient.

Remark 1. This notion of near invariance is analogous to the one introduced in [8] for Markov diffusion systems. However, in the present paper arbitrary parameters $\alpha$ are allowed, not just the maximal amplitude of a random disturbance as in [8]. In the latter case, it is clear that existence of a sequence $\alpha_{n} \searrow \alpha_{0}$ with property (ii) implies that this property holds for all $\alpha>\alpha_{0}$ with $\alpha-\alpha_{0}$ small. For a locally transient set we require that the systems with $\alpha_{n}$ leave a neighborhood $W$ of $A$. Note that the system with $\alpha_{n}$ may return to $W$ at some later time, hence there may exist a stationary measure whose support intersects or even includes $W$. Hence transient as well as intermittent bifurcations in the sense of [21] are included.

We note the following characterization of locally transient, nearly invariant sets. 
Proposition 2. A closed set $A \subset M$ is a locally transient, nearly invariant set for $\alpha_{n} \rightarrow \alpha_{0}$ at $x_{0} \in \operatorname{int} A$, if and only if there is a neighborhood $W$ of $A$ with the following properties: (i) for all $n \in \mathbb{N}$ the first exit time of $x_{0}$ from $W$ satisfies $\chi_{\alpha_{n}}\left(x_{0}, \omega, W\right)<\infty$ with positive probability; (ii) for all $x \in A$ one has $\chi_{\alpha_{n}}(x, \omega, A) \rightarrow \infty$ almost surely for $n \rightarrow \infty$ and $\chi_{\alpha_{0}}(x, \omega, A)=\infty$.

Proof. If $\chi_{\alpha}\left(x_{0}, \omega, W\right)<\infty$, then $\chi_{\alpha}\left(x_{0}, \omega, A\right)<\infty$. Hence a set satisfying properties (i) and (ii) is a nearly invariant set and clearly it is locally transient. The converse is obvious.

The following result characterizes locally transient, nearly invariant sets by properties of the associated discrete time control systems.

TheOREM 4. Suppose that hypotheses (H1)-(H3) are satisfied for the family $f_{\alpha}, \alpha \in I$, of random diffeomorphisms. Let $x_{0} \in \operatorname{int} A$ for some closed set $A \subset M$ and consider a sequence $\alpha_{n} \rightarrow \alpha_{0}$ in $I$. Then the set $A$ is a locally transient, nearly invariant set for $\left(\alpha_{n}\right)$ in $x_{0}$ if and only if $A$ is positively invariant for $\alpha_{0}$ and there is a neighborhood $W$ of $A$ such that

$$
\operatorname{int}\left(\mathcal{O}^{\alpha_{n},+}\left(x_{0}\right) \backslash \operatorname{cl} W\right) \neq \emptyset \text { for each } n \in \mathbb{N} .
$$

Proof. First we show that positive invariance of $A$ and continuity imply property (ii) of Proposition 2. Positive invariance of $A$ shows that $\chi_{\alpha_{0}}(x, \omega, A)=\infty$ almost surely. Now assume contrary to the other assertion that there are $x \in A$ and a positive time $N$ such that $P_{x}\left(\chi_{\alpha_{n}}(x, \omega, A)<N\right)>0$ for all $n$. Thus (taking a corresponding realization of the noise) there are controls $u^{n}$ with $f_{\alpha_{n}}^{N}\left(x, u^{n}\right) \notin A$. Then there is a subsequence $\left(u^{n_{k}}\right)$ such that $u^{n_{k}} \rightarrow u_{*} \in \mathcal{U}$. By continuity it follows that $f_{\alpha_{n_{k}}}^{N}\left(x, u^{n_{k}}\right) \rightarrow f_{\alpha_{0}}^{N}\left(x, u_{*}\right)$. Then $f_{\alpha_{0}}^{N}\left(x, u_{*}\right)$ is in the closure of the complement of $A$. By positive invariance of $A$ it follows that $f_{\alpha_{0}}^{N}\left(x, u_{*}\right) \in \partial A$. Again using continuity with respect to the initial point, one sees that this contradicts positive invariance of $A$.

Next we prove that assumption (5) implies property (i) of Proposition 2, i.e., that $P_{x_{0}}\left(\chi_{\alpha_{n}}(x, \omega, W)<\infty\right)>0$ for all $n$. Pick $\alpha_{n}$, then there are some open set $V \subset \operatorname{int}\left(\mathcal{O}^{\alpha_{n},+}\left(x_{0}\right) \backslash \operatorname{cl} W\right)$, a positive time $N<\infty$, and a control $u^{0} \in \mathcal{U}$ such that $f_{\alpha}^{N}\left(x_{0}, u^{0}\right) \in V$. By continuous dependence of the solutions on $u$, there is $\varepsilon>0$ such that for all $u$ in $V_{\varepsilon}^{N}\left(u^{0}\right):=\left\{u:\{0,1, \ldots, N\} \rightarrow \Delta\right.$ with $\left\|u_{k}-u_{k}^{0}\right\|<\varepsilon$ for all $\left.k\right\}$ one has $\varphi_{\alpha}\left(N, x_{0}, u\right) \in V$. Lemma 5 implies that $\nu^{N}\left(V_{\varepsilon}^{N}\left(u^{0}\right)\right)>0$. We obtain

$$
P_{x_{0}}\left(\chi_{\alpha_{n}}\left(x_{0}, \omega, W\right)<\infty\right) \geq P_{x_{0}}\left(\chi_{\alpha_{n}}\left(x_{0}, \omega, W\right) \leq T\right) \geq \nu^{N}\left(V_{\varepsilon}^{N}\left(u^{0}\right)\right)>0 .
$$

For the converse implication assume that $A$ is a locally transient, nearly invariant set in $x_{0} \in \operatorname{int} A$ for $\left(\alpha_{n}\right)$. Then, for a neighborhood $W_{1}$ of $A$ with $\operatorname{cl} W_{1} \subset W$, one has $\chi_{\alpha_{n}}\left(x_{0}, \omega, \operatorname{cl} W_{1}\right)<\infty$ with positive probability for all $n$. Thus for every $n$ there is a realization $u_{n}$ of $\omega$ and a time $N$ such that

$$
f_{\alpha_{n}}^{N}\left(x_{0}, u_{n}\right) \notin \mathrm{cl} W_{1} .
$$

Thus $f_{\alpha_{n}}^{N}\left(x_{0}, u_{n}\right) \in \mathcal{O}^{\alpha_{n},+}\left(x_{0}\right) \backslash \operatorname{cl} W$. Lemma 1 and hypotheses (H1) and(H2) imply that

$$
\mathcal{O}^{\alpha_{n},+}\left(x_{0}\right) \subset \operatorname{cl} \operatorname{int} \mathcal{O}^{\alpha_{n},+}\left(x_{0}\right) .
$$

Since $A$ is closed, we see that for every $n$ condition (5) holds. It remains to show that the set $A$ is positively invariant for $\alpha_{0}$. This follows from $\chi_{\alpha_{0}}(x, \omega, A)=\infty$ 
almost surely. In fact, if $A$ is not positively invariant, we obtain a contradiction using the same reasoning as above in the proof that (5) implies property (i) of Proposition 2.

The following corollary is an analogue of Theorem 3.2 in [8].

COROllary 1. Suppose that the assumptions of Theorem 4 are satisfied. Then the set $A$ is nearly invariant in $x_{0}$ for $\left(\alpha_{n}\right)$ if and only if the set $A$ is positively invariant for $\alpha_{0}$ and

$$
\operatorname{int}\left(\mathcal{O}^{\alpha_{n},+}\left(x_{0}\right) \backslash A\right) \neq \emptyset \text { for each } n \in \mathbb{N} .
$$

Proof. This follows as in Theorem 4.

Next we show that invariant control sets, which lose their invariance at $\alpha_{0}$, are locally transient, nearly invariant sets. This requires a generalization of Gayers continuous time result [18, Corollary 24], stating that loss of invariance for increasing control range (determined by $\alpha$ ) implies a discontinuity of $\alpha \mapsto \operatorname{cl} D^{\alpha}$.

Proposition 3. Let $D^{\alpha_{0}}$ be an invariant control set.

(i) There is $\varepsilon_{0}>0$ such that for all $\alpha$ with $\left|\alpha-\alpha_{0}\right|<\varepsilon_{0}$ there is a unique control set $D^{\alpha}$ with the following property: For all compact subsets $K \subset \operatorname{core} D^{\alpha_{0}}$ there is $\varepsilon_{1} \in\left(0, \varepsilon_{0}\right)$ such that $K \subset \operatorname{core} D^{\alpha}$ for all $\alpha$ with $0<\alpha-\alpha_{0}<\varepsilon_{1}$.

(ii) Suppose that the map $\alpha \mapsto \operatorname{cl} D^{\alpha}$ is continuous at $\alpha=\alpha_{0}$. Then there is $\varepsilon_{2} \in\left(0, \varepsilon_{1}\right)$ such that for all $\alpha$ with $0<\alpha-\alpha_{0}<\varepsilon_{2}$ the $D^{\alpha}$ are invariant control sets.

Proof. (i) This follows from the lower semicontinuity property in Theorem 2.

(ii) By Lemma 4 we have to show that the boundary of $D^{\alpha}$ coincides with its entrance boundary. Fix a compact neighborhood $K \subset \operatorname{core} D^{\alpha_{0}}$ of $x_{0} \in \operatorname{core} D^{\alpha_{0}}$ and choose $\varepsilon_{0}>0$ according to (i). For each $x \in D^{\alpha_{0}}$ there is $u_{x} \in \mathcal{U}$ and $t_{x}>0$ such that $\varphi_{\alpha_{0}}\left(t_{x}, x, u_{x}\right)=x_{0}$. Thus there are $\varepsilon_{x} \in(0, \varepsilon)$ and an open neighborhood $V_{x}$ of $x$ such that $\varphi_{\alpha}\left(t_{x}, V_{x}, u_{x}\right) \subset K$ for all $\alpha$ with $0<\alpha-\alpha_{0}<\varepsilon_{x}$. Since the invariant control set $D^{\alpha_{0}}$ is compact, finitely many of the neighborhoods $V_{x}$ cover $D^{\alpha_{0}}$. Since the map $\alpha \mapsto \operatorname{cl} D^{\alpha}$ is continuous at $\alpha=\alpha_{0}$, one finds that for all points $x \in \operatorname{cl} D^{\alpha}$ with $0<\alpha-\alpha_{0}<\varepsilon_{2}$ that

$$
\emptyset \neq \mathcal{O}^{\alpha,+}(x) \cap K \subset \mathcal{O}^{\alpha,+}(x) \cap \operatorname{core} D^{\alpha} .
$$

In particular, this is true for all points in the boundary $\partial D^{\alpha}$, i.e., $\partial D^{\alpha}=\partial^{e n} D^{\alpha}$, and it follows that these $D^{\alpha}$ are invariant control sets.

We note the following consequence at discontinuity points.

Proposition 4. Let $D^{\alpha_{0}}$ be an invariant control set and suppose that the map $\alpha \mapsto \operatorname{cl} D^{\alpha}$ is discontinuous at $\alpha=\alpha_{0}$. Then there are $\alpha_{n} \rightarrow \alpha_{0}$ such that for every compact subset $K \subset \operatorname{int} D^{\alpha_{0}}$ there is a neighborhood $W$ of $D^{\alpha_{0}}$ such that $\operatorname{int}\left(\mathcal{O}^{\alpha_{n},+}\left(x_{0}\right) \backslash \mathrm{cl} W\right) \neq \emptyset$ for all $n$ and all $x_{0} \in K$.

Proof. Discontinuity in the Hausdorff metric means that for $\alpha \rightarrow \alpha_{0}$

$$
\text { (i) } \sup _{x \in D^{\alpha_{0}}} \mathrm{~d}\left(x, \operatorname{cl} D^{\alpha}\right) \not 0 \text { or (ii) } \sup _{x \in D^{\alpha}} \mathrm{d}\left(x, D^{\alpha_{0}}\right) \nrightarrow 0 \text {. }
$$

Case (i) cannot occur, due to Theorem 2 establishing lower semicontinuity of control sets. In case (ii), one finds $\varepsilon>0, \alpha_{n} \rightarrow \alpha_{0}$, and $x_{n} \in D^{\alpha_{n}}$ such that $\mathrm{d}\left(x_{n}, D^{\alpha_{0}}\right) \geq \varepsilon$. 
By Theorem 2 again and by approximate controllability in $D^{\alpha_{n}}$, we may assume that $x_{n} \in \mathcal{O}^{\alpha_{n},+}\left(x_{0}\right)$ for all $x_{0} \in K \subset \operatorname{int} D^{\alpha_{0}}$ for all $n \in \mathbb{N}$. Hence there is a neighborhood $W$ of $D^{\alpha_{0}}$ such that $\operatorname{int}\left(\mathcal{O}^{\alpha_{n},+}\left(x_{0}\right) \backslash \operatorname{cl} W\right) \neq \emptyset$ for all $x_{0} \in K$.

The following theorem shows the announced result on locally transient, nearly invariant sets.

THEOREM 5. Assume that the family of random diffeomorphisms $f_{\alpha}, \alpha \in I$, satisfies hypotheses (H1)-(H3). Let $D^{\alpha_{0}}$ be an invariant control set of the associated control system (2) and suppose that the map $\alpha \mapsto \mathrm{cl} D^{\alpha}$ from Proposition 3 is not continuous in $\alpha=\alpha_{0}$. This holds in particular, if there are $\beta_{n} \rightarrow \alpha_{0}$ such that the control sets $D^{\beta_{n}}$ are not invariant. Then $D^{\alpha_{0}}$ is a locally transient, nearly invariant set for a sequence $\alpha_{n} \rightarrow \alpha_{0}$.

Proof. If there are $\beta_{n} \rightarrow \alpha_{0}$ such that the control sets $D^{\beta_{n}}$ are not invariant, discontinuity in the Hausdorff metric follows from Proposition 3 (ii). Let $x_{0} \in$ $\operatorname{int} D^{\alpha_{0}}$. By Proposition 4 (ii) there are $\alpha_{n} \rightarrow \alpha_{0}$ and a neighborhood $W$ of $D^{\alpha_{0}}$ such that $\operatorname{int}\left(\mathcal{O}^{\alpha_{n},+}\left(x_{0}\right) \backslash \mathrm{cl} W\right) \neq \emptyset$ for all $n$. Since $D^{\alpha_{0}}$ is a control set, this holds for all $x_{0} \in \operatorname{int} D^{\alpha_{0}}$ and since $D^{\alpha_{0}}$ is an invariant control set, it is positively invariant for $\alpha_{0}$. Hence by Theorem 4 (ii) the set $D^{\alpha_{0}}$ is a locally transient, nearly invariant set in $x_{0}$ for a sequence $\alpha_{n} \rightarrow \alpha_{0}$.

Finally we consider escape times from a nearly invariant, locally transient set $A$. We restrict ourselves to the situation of Theorem 5 . The following result shows that exit times are finite and polynomially bounded below.

Theorem 6. Consider a family $f_{\alpha}, \alpha \in I$, of random diffeomorphisms in $\mathcal{R}^{\infty}(M)$ satisfying hypotheses (H1)-(H3) with the parameter $\alpha$ from an open interval I. Let $\mu_{\alpha_{0}}$ be an ergodic stationary measure of $f_{\alpha_{0}}$ for some $\alpha_{0} \in I$. Then the support of $\mu_{\alpha_{0}}$ is an invariant control set $D^{\alpha_{0}}$ and there is a neighborhood $W$ of $D^{\alpha_{0}}$ such that no other stationary measure has support intersecting $\mathrm{cl} W$. Assume that there are $\beta_{n} \rightarrow \alpha_{0}$ such that the control sets $D^{\beta_{n}}$ from Proposition 3 are not invariant.

(i) Then $D^{\alpha_{0}}$ is a locally transient, nearly invariant set for a sequence $\alpha_{n} \rightarrow \alpha_{0}$ and for all $k \in \mathbb{N}$ there constants $c_{k}>0$ with

$$
\int_{W} \int_{\Delta^{\mathbb{N}}} \chi_{\alpha_{n}}(x, \omega, W) \mathrm{d} \nu^{\infty}(\omega) \mathrm{d} m(x) \geq c_{k}\left|\alpha_{n}-\alpha_{0}\right|^{-k}
$$

(ii) there is a neighborhood $V$ of $D^{\alpha_{0}}$ such that for every compact subset $\emptyset \neq$ $K \subset \operatorname{int} D^{\alpha_{0}}$ there are times $T_{n}>0$ such that for all $x \in K$

$$
\chi_{\alpha_{n}}(x, \omega, V) \leq T_{n} \text { with positive probability. }
$$

Proof. By Proposition 3, the support of each ergodic stationary measure is an invariant control set $D^{\alpha_{0}}$. Then existence of the neighborhood $W$ follows, since there are only finitely many invariant (and compact) control sets. Now Theorem 1 implies the existence of constants $c_{k}>0$ such that $(7)$ holds. It remains to show the existence of the upper bounds $T_{n}$ in (8). Theorem 5 shows that $D^{\alpha_{0}}$ is a locally transient, nearly invariant set for a sequence $\alpha_{n} \rightarrow \alpha_{0}$. Hence there is a neighborhood $V_{1}$ such that $\operatorname{int}\left(\mathcal{O}^{\alpha_{n},+}\left(x_{0}\right) \backslash \mathrm{cl} V_{1}\right) \neq \emptyset$ follows. Choose a compact set $K \subset \operatorname{int} D^{\alpha_{0}}$ with nonvoid interior. By Theorem 4, there is a neighborhood $V$ of $D^{\alpha_{0}}$ such that $\operatorname{int}\left(\mathcal{O}^{\alpha_{n},+}\left(x_{0}\right) \backslash \mathrm{cl} V\right) \neq \emptyset$ for all $n$ and all $x_{0} \in K$. Then there is a tube around the corresponding trajectory leaving $\mathrm{cl} V$. Hence the first exit time 
from all points in $K$ is bounded above with positive probability and the assertion follows.

Remark 2. In particular, Theorem 6 gives estimates for escape times for problems, where the bifurcation parameter $\alpha$ is taken as the maximal amplitude of the random perturbations, i.e., for families of random diffeomorphisms of the form

$$
f_{\alpha}(x, \omega):=f(x, \alpha \omega),
$$

where $f: M \times \mathbb{R}^{d} \rightarrow M$ and $\Delta$ is the unit ball in $\mathbb{R}^{d}$.

Remark 3. The question if results similar to Theorem 6 hold for more general locally transient, nearly invariant sets, remains open.

\section{REFERENCES}

[1] F. Albertini and E. D. Sontag, Discrete-time transitivity and accessibility: Analytic systems, SIAM J. Control Optim., 31 (1993), pp. 1599-1622.

[2] L. Arnold and W. Kliemann, Qualitative theory of stochastic systems, in Probabilistic Analysis and Related Topics, Vol. 3, A.T. Bharucha-Reid, ed., Academic Press, New York, 1983, pp. 1-79.

[3] — On unique ergodicity for degenerate diffusions, Stochastics, 21 (1987), pp. $41-61$.

[4] C. BAuER, Limit behaviour and control sets of discrete-time systems, Random and Comp. Dyn., 4 (1996), pp. 269 299.

[5] A. Bovier, M. Eckhoff, V. Gayrard, and M. Klein, Metastability in reversible diffusion processes. I. Sharp asymptotics for capacities and exit times, J. Eur. Math. Soc. (JEMS), 6 (2004), p. 399-424.

[6] A. Bovier, V. Gayrard, And M. Klein, Metastability in reversible diffusion processes. II. Precise asymptotics for small eigenvalues, J. Eur. Math. Soc. (JEMS), 7 (2005), p. 69-99.

[7] O. Class, Lineare Extensionen und Diskrete Kontrollsysteme. Diplomarbeit, Universität Augsburg, 2003.

[8] F. Colonius, T. Gayer, and W. Kliemann, Near invariance for Markov diffusion systems, SIAM J. Applied Dyn. Systems, 7 (2008), pp. 79-107.

[9] F. Colonius and W. Kliemann, The Dynamics of Control, Birkhäuser, Boston, 2000.

[10] M. Dellnitz and O. Junge, Almost invariant sets in Chuaś circuit, Internat.J.Bifur.Chaos Appl.Sci.Engrg, 7 (1997), pp. 2475-2485.

[11] _ Set oriented numerical methods for dynamical systems, in Handbook of Dynamical Systems, Volume 2, B. Fiedler, ed., Elsevier, 2002, ch. 5, pp. 221264.

[12] M. Dellnitz, M. H. von Molo, P. Metzner, R. Preis, and C. Schütte, Graph algorithms for dynamical systems, in Analysis, Modeling and Simulation of Multiscale Problems, A. Mielke, ed., Springer-Verlag, 2006, pp. 619645. 
[13] P. Deuflhard, W. Huisinga, A. Fischer, and C. Schütte, Identification of almost invariant aggregates in reversible nearly uncoupled markov chains, Lin. Alg. Appl., 315 (2000), pp. 39-59.

[14] M. Freidlin and A. Wentzell, Random Perturbations of Dynamical Systems, Springer-Verlag, 1984.

[15] G. Froyland, Statistically optimal almost-invariant sets, Phys. D, 200 (2005), pp. 205219.

[16] G. Froyland and M. Dellnitz, Detecting and locating near-optimal almost -invariant sets and cycles, SIAM J. Sci. Comp., 24 (2004), pp. 1839-1863.

[17] T. Gayer, On Markov chains and the spectra of corresponding FrobeniusPerron operators, Stochastics and Dynamics, 1 (2001), pp. 477-491.

[18] _ Control sets and their boundaries under parameter variation, J. Diff. Equations, 201 (2004), pp. 177-200.

[19] C. Schütte, W. Huisinga, and P. Deuflhard, Transfer operator approach to conformational dynamics in biomolecular systems, in Ergodic Theory, Analysis, and Effi cient Simulation of Dynamical Systems, B. Fiedler, ed., Springer-Verlag, New York, 2001, p. 191-223.

[20] C. Schütte, W. Huisinga, And S. Meyn, Phase transitions \& metastability in Markovian and molecular systems, Ann. Appl. Probab., 14 ((2004)), pp. $419-458$.

[21] H. Zmarrou And A. J. Homburg, Bifurcations of stationary measures of random diffeomorphisms, Ergodic Theory and Dynamical Systems, 27 (2007), pp. $1651-1692$. 